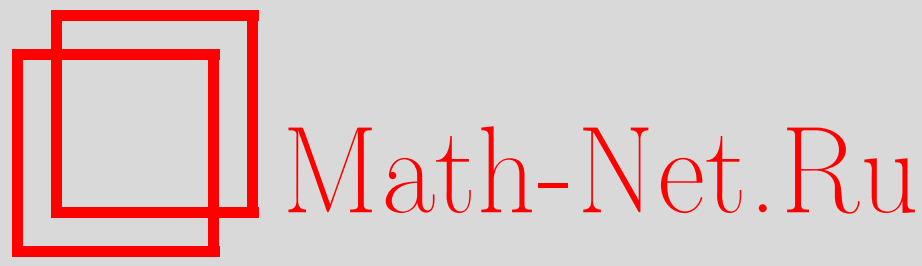

В. А. Белавин, Модулярные интегралы в минимальной лиувиллевской супергравитации, ТМФ, 2009, том 161, номер 1, 46-62

DOI: https://doi.org/10.4213/tmf6418

Использование Общероссийского математического портала Math-Net.Ru подразумевает, что вы прочитали и согласны с пользовательским соглашением http://www.mathnet.ru/rus/agreement

Параметры загрузки:

IP: 18.208 .226 .222

26 апреля 2023 г., 03:04:23

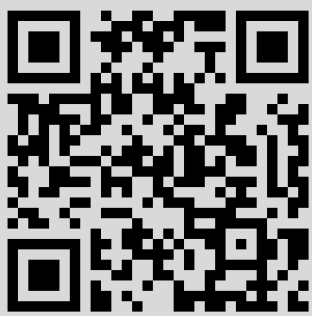




\title{
МОДУЛЯРНЫЕ ИНТЕГРАЛЫ В МИНИМАЛЬНОЙ ЛИУВИЛЛЕВСКОЙ СУПЕРГРАВИТАЦИИ
}

\begin{abstract}
Численно найден четырехточечный интеграл минимальной лиувиллевской супергравитации на сфере. Процедура интегрирования основана на эффективной эллиптической параметризации пространства модулей. Анализ выполнен для нескольких различных гравитационных четырехточечных амплитуд. Результаты согласуются с аналитическими результатами, полученными недавно с использованием высших суперсимметричных уравнений Лиувилля.
\end{abstract}

Ключевые слова: суперконформная теория поля, суперструны, двумерная лиувиллевская супергравитация.

\section{1. ВВЕДЕНИЕ}

Континуальная формулировка теории некритических струн эквивалентна двумерной квантовой гравитации, взаимодействующей с некоторой критической материей, т.е. с материей, описываемой конформной теорией поля $\mathcal{M}_{c}$. Простой отклик конформных теорий на скейлинг метрики приводит к универсальному виду эффективного действия индуцированной гравитации, которая называется лиувиллевской гравитацией (ЛГ) [1]. В силу особенностей двумерной метрической геометрии возникают многочисленные технические упрощения. Таким образом, ЛГ является, вероятно, простейшим примером квантовой гравитации, но при этом ей присущи те же основные вопросы интерпретации, а потому она может считаться полезной и заслуживающей изучения. Задачи о правильном выборе наблюдаемых и о вычислении соответствующих корреляционных функций имеют первостепенную важность для любой квантовой теории. В области исследования ЛГ в последние годы произошел существенный прогресс. Полученные в работе [2] высшие уравнения движения (ВУД) в лиувиллевской теории поля позволили вычислить четырехточечные корреляторы в ЛГ [3], [4]. Результаты были проверены путем сравнения с вычислением в рамках независимого подхода к двумерной квантовой гравитации, обычно

${ }^{*}$ Институт теоретической и экспериментальной физики, Москва, Россия; Laboratoire de Physique Théorique et Astroparticules, Université Montpellier II, Montpellier, France.

E-mail: vlbelavin@yandex.ru 
называемого матричными моделями (см., например, [5] и приведенную там библиографию). Более того, на основе этих результатов можно значительно продвинуться в понимании соответствия между этими двумя подходами [6], хотя полной картины соотношения между двумя методами до сих пор нет.

С точки зрения теории струн наиболее интересным является вопрос о конструкции двумерной квантовой гравитации в суперпространстве. Первая возможность обобщения предоставляется $N=1$ суперсимметрией. Здесь также недавно был достигнут значительный прогресс. Исследование мотивировалось рядом работ по суперсимметричной теории поля Лиувилля, в которых было достигнуто глубокое понимание свойств конформных блоков (являющихся базисными элементами всякой конформной теории поля [7]). Это позволило не рассматривать корреляции, содержащие только специальные вырожденные возбуждения [8]. Другим важным результатом стало открытие ВУД в суперсимметричной теории поля Лиувилля [9]. Все эти результаты послужили отправной точкой для более глубокого исследования лиувиллевской супергравитации (ЛСГ). В работе [10] была выяснена структура физических полей в секторе Неве-Шварца в ЛСГ, а также было явно записано общее выражение для $n$-точечных корреляционных чисел на сфере в терминах интегралов по пространству модулей. При этом суперсимметричные ВУД $N=1$ суперсимметричной теории поля Лиувилля и анализ структуры основного суперкольца физических состояний (и его логарифмического аналога) позволяют вывести явные аналитические выражения для четырехточечных корреляторов. Поскольку о суперсимметричных матричных моделях на данный момент известно немного, не существует независимых результатов, аналогичных полученным в работе [10]. В этих условиях желательна дополнительная проверка справедливости полученных в [10] результатов. Данная работа посвящена непосредственному вычислению четырехточечных корреляционных чисел в минимальной ЛСГ.

Работа построена следующим образом. В разделах 2 и 3 мы приводим все необходимые сведения о предмете. В разделе 4 рассмотрены два примера четырехточечных интегралов. Мы сводим выражения к некоторому числу интегралов по фундаментальной области модулярной группы, причем подынтегральные выражения представляют собой произведения различных корреляционных функций как в материальном, так и в лиувиллевском секторе. Эллиптическое преобразование является предметом раздела 5. Численные результаты представлены в разделе 6 . Некоторые подробности, опущенные в основном тексте, приводятся в приложении.

\section{2. МИНИМАЛЬНАЯ ЛИУВИЛЛЕВСКАЯ СУПЕРГРАВИТАЦИЯ}

В рамках так называемого подхода Давида-Дистлера-Каваи [11]-[13] ЛСГ представляет собой тензорное произведение суперконформной материи, суперсимметричной системы Лиувилля и супердуховой системы:

$$
A_{\text {лСГ }}=A_{\mathrm{SM}}+A_{\mathrm{SL}}+A_{\mathrm{SG}},
$$


причем взаимодействие осуществляется в силу соотношения для центральных зарядов:

$$
c_{\mathrm{SM}}+c_{\mathrm{SL}}+c_{\mathrm{SG}}=0,
$$

а также за счет конструкции физических полей.

Суперконформная алгебра имеет вид

$$
\begin{aligned}
{\left[L_{n}, L_{m}\right] } & =(n-m) L_{n+m}+\frac{\hat{c}}{8}\left(n^{3}-n\right) \delta_{n,-m}, \\
\left\{G_{r}, G_{s}\right\} & =2 L_{r+s}+\frac{\hat{c}}{2}\left(r^{2}-\frac{1}{4}\right) \delta_{r,-s}, \\
{\left[L_{n}, G_{r}\right] } & =\left(\frac{1}{2} n-r\right) G_{n+r},
\end{aligned}
$$

где $r, s \in \mathbb{Z}+1 / 2$ в секторе Неве-Шварца, $r, s \in \mathbb{Z}$ в секторе Рамона.

Центральный заряд суперсимметричной теории поля Лиувилля равен

$$
\widehat{c}_{\mathrm{SL}}=1+2 Q^{2}
$$

где параметр “фонового заряда" $Q$ связан с основным квантовым параметром $b$ в суперсимметричной теории поля Лиувилля как $Q=b^{-1}+b$. Поля принадлежат к представлениям суперконформной алгебры со старшим весом. Базисные поля, которые интересуют нас в данной работе, принадлежат к примарному супермультиплету $\left(V_{a}, Y_{a}, \bar{Y}_{a}, W_{a}\right)$ с младшей компонентой $V_{a}$, имеющей конформную размерность

$$
\Delta_{a}^{\mathrm{L}}=\frac{a(Q-a)}{2}
$$

где $a$ - непрерывный (комплексный) параметр, а остальные компоненты примарного супермультиплета имеют вид

$$
Y_{a}=G_{-1 / 2}^{\mathrm{L}} V_{a}, \quad \bar{Y}_{a}=\bar{G}_{-1 / 2}^{\mathrm{L}} V_{a}, \quad W_{a}=\bar{G}_{-1 / 2}^{\mathrm{L}} G_{-1 / 2}^{\mathrm{L}} V_{a} .
$$

Здесь и далее используются индексы M, L, и G для указания на суперконформные генераторы в материальном, лиувиллевском и духовом секторах. Генераторы без индекса, указывающего на сектор, относятся к полной супералгебре Вирасоро.

При некоторых специальных значениях параметра $a=a_{m, n}$ в модуле Верма над $V_{a_{m, n}}=V_{m, n}$ появляется один сингулярный вектор на уровне $m n / 2$ [8]. Тогда

$$
a_{m, n}=\frac{Q}{2}-\lambda_{m, n}
$$

где $(m, n)$ - пара положительных целых чисел $(m-n \in 2 \mathbb{Z})$ и $\lambda_{m, n}=\left(m b^{-1}+n b\right) / 2$.

Базисное суперсимметричное операторное разложение Лиувилля имеет вид [14] (для краткости мы пишем $\Delta=\Delta_{Q / 2+i P}$ и $\Delta_{i}=\Delta_{a_{i}}$ )

$$
\begin{aligned}
& V_{a_{1}}(x) V_{a_{2}}(0)= \\
& \quad=\int^{\prime} \frac{d P}{4 \pi}(x \bar{x})^{\Delta-\Delta_{1}-\Delta_{2}}\left(\mathbb{C}_{a_{1}, a_{2}}^{Q / 2+i P}\left[V_{Q / 2+i P}(0)\right]_{\mathrm{ee}}+\tilde{\mathbb{C}}_{a_{1}, a_{2}}^{Q / 2+i P}\left[V_{Q / 2+i P}(0)\right]_{\text {oo }}\right) .
\end{aligned}
$$


Это операторное произведение является непрерывным и включает в себя интегрирование по "импульсу" $P$. В разложении (8) $\left[V_{p}\right]_{\text {ее,оо }}$ обозначает вклад в операторное произведение примарного поля $V_{p}$ и его "четных" и "нечетных" суперконформных потомков. Как обычно, штрих у интеграла указывает на возможные дискретные слагаемые; в данной работе мы рассматриваем только такую область $b$, в которой подобные дополнительные слагаемые не возникают и интеграл можно понимать буквально. Все остальные операторные произведения двух произвольных локальных полей в суперсимметричной теории поля Лиувилля можно вывести из разложения (8). Базисные структурные константы $\mathbb{C}_{a_{1} a_{2}}^{Q / 2+i P}$ и $\tilde{\mathbb{C}}_{a_{1}, a_{2}}^{Q / 2+i P}$ из (8) были вычислены в работах [15], [16] с использованием бутстрапного подхода, они имеют следующий вид (здесь $a$ обозначает $a_{1}+a_{2}+a_{3}$ ):

$$
\begin{aligned}
& \mathbb{C}_{a_{1} a_{2}}^{Q-a_{3}}=\left(\pi \mu \gamma\left(\frac{Q b}{2}\right) b^{1-b^{2}}\right)^{(Q-a) / b} \frac{\Upsilon_{\mathrm{R}}(b) \Upsilon_{\mathrm{NS}}\left(2 a_{1}\right) \Upsilon_{\mathrm{NS}}\left(2 a_{2}\right) \Upsilon_{\mathrm{NS}}\left(2 a_{3}\right)}{2 \Upsilon_{\mathrm{NS}}(a-Q) \Upsilon_{\mathrm{NS}}\left(a_{1+2-3}\right) \Upsilon_{\mathrm{NS}}\left(a_{2+3-1}\right) \Upsilon_{\mathrm{NS}}\left(a_{3+1-2}\right)}, \\
& \tilde{\mathbb{C}}_{a_{1} a_{2}}^{Q-a_{3}}=-\left(\pi \mu \gamma\left(\frac{Q b}{2}\right) b^{1-b^{2}}\right)^{(Q-a) / b} \frac{i \Upsilon_{\mathrm{R}}(b) \Upsilon_{\mathrm{NS}}\left(2 a_{1}\right) \Upsilon_{\mathrm{NS}}\left(2 a_{2}\right) \Upsilon_{\mathrm{NS}}\left(2 a_{3}\right)}{\Upsilon_{\mathrm{R}}(a-Q) \Upsilon_{\mathrm{R}}\left(a_{1+2-3}\right) \Upsilon_{\mathrm{R}}\left(a_{2+3-1}\right) \Upsilon_{\mathrm{R}}\left(a_{3+1-2}\right)},
\end{aligned}
$$

где использовано удобное обозначение [17] для специальных функций

$$
\begin{aligned}
\Upsilon_{\mathrm{NS}}(x) & =\Upsilon_{b}\left(\frac{x}{2}\right) \Upsilon_{b}\left(\frac{x+Q}{2}\right) \\
\Upsilon_{\mathrm{R}}(x) & =\Upsilon_{b}\left(\frac{x+b}{2}\right) \Upsilon_{b}\left(\frac{x+b^{-1}}{2}\right)
\end{aligned}
$$

выражающихся в терминах ипсилон-функции $\Upsilon_{b}$, которая является стандартным элементом в лиувиллевской теории поля (см. [18], [19]).

Из-за условия баланса центральных зарядов (2) центральный заряд в материальном секторе выражается через тот же базисный параметр $b$ :

$$
\widehat{c}_{\mathrm{SM}}=1-2 q^{2}
$$

где $q=b^{-1}-b$. Пусть $\left(\Phi_{\alpha}, \chi_{\alpha}, \bar{\chi}_{\alpha}, \Psi_{\alpha}\right)$ обозначает примарный мультиплет в материальном секторе с размерностью младшей компоненты $\Phi_{a}$, равной

$$
\Delta_{\alpha}^{\mathrm{M}}=\frac{\alpha(q-\alpha)}{2}
$$

Супердуховая система (см., например, [20]-[22]) описывается свободной суперконформной теорией поля с центральным зарядом $c_{\mathrm{SG}}=-10$. Фермионная часть супердуховой системы включает пару антикоммутирующих полей $(b, c)$ со спинами $(2,-1)$, а бозонная часть - пару бозонных полей $(\beta, \gamma)$ со спинами $(3 / 2,-1 / 2)$. Формальное поле (см. [10]) вида $\delta(\gamma(0))$, имеющее размерность $1 / 2$, играет существенную роль в построении гравитационных амплитуд. 


\section{3. ФИЗИЧЕСКИЕ ПОЛЯ И КОРРЕЛЯЦИОННЫЕ ЧИСЛА}

Физические поля образуют пространство классов когомологий по отношению к нильпотентному БРСТ-заряду

$$
\mathbb{Q}=\sum_{m}:\left[L_{m}^{\mathrm{M}+\mathrm{L}}+\frac{1}{2} L_{m}^{\mathrm{G}}\right] c_{-m}:+\sum_{r}:\left[G_{r}^{\mathrm{M}+\mathrm{L}}+\frac{1}{2} G_{r}^{\mathrm{G}}\right] \gamma_{-r}:-\frac{1}{4} c_{0} .
$$

В данной работе мы имеем дело с корреляторами физических полей двух типов:

$$
\mathbb{W}_{a}(z, \bar{z})=\mathbb{U}_{a}(z, \bar{z}) \cdot c(z) \bar{c}(\bar{z}) \cdot \delta(\gamma(z)) \delta(\bar{\gamma}(\bar{z}))
$$

И

$$
\tilde{\mathbb{W}}_{a}(z, \bar{z})=\left(\bar{G}_{-1 / 2}^{\mathrm{M}+\mathrm{L}}+\frac{1}{2} \bar{G}_{-1 / 2}^{\mathrm{G}}\right)\left(G_{-1 / 2}^{\mathrm{M}+\mathrm{L}}+\frac{1}{2} G_{-1 / 2}^{\mathrm{G}}\right) \mathbb{U}_{a}(z, \bar{z}) \cdot \bar{c}(\bar{z}) c(z),
$$

где

$$
\mathbb{U}_{a}(z, \bar{z})=\Phi_{a-b}(z, \bar{z}) V_{a}(z, \bar{z}) .
$$

Здесь параметр а может принимать значения общего положения. Общий вид $n$-точечного корреляционного числа на сфере для этих наблюдаемых [10] таков:

$$
I_{n}\left(a_{1}, \ldots, a_{n}\right)=\prod_{i=4}^{n} \int d^{2} z_{i}\left\langle\bar{G}_{-1 / 2} G_{-1 / 2} \mathbb{U}_{a_{i}}\left(z_{i}\right) \tilde{\mathbb{W}}_{a_{3}}\left(z_{3}\right) \mathbb{W}_{a_{2}}\left(z_{2}\right) \mathbb{W}_{a_{1}}\left(z_{1}\right)\right\rangle .
$$

Дополнительное “дискретное" физическое состояние возникает, когда представление в материальном секторе вырождается:

$$
\mathbb{O}_{m, n}(z, \bar{z})=\bar{H}_{m, n} H_{m, n} \Phi_{m, n}(z, \bar{z}) V_{m, n}(z, \bar{z}) \text {. }
$$

Операторы $H_{m, n}$ составлены из генераторов суперконформной алгебры Вирасоро и определяются однозначно по модулю точных членов. Более того, если ввести логарифмические аналоги дискретных состояний $\mathbb{O}_{m, n}$,

$$
\mathbb{O}_{m, n}^{\prime}=\bar{H}_{m, n} H_{m, n} \Phi_{m, n} V_{m, n}^{\prime}
$$

то имеются важные соотношения [10]

$$
\overline{\mathbb{Q}} \mathbb{Q} \mathbb{O}_{m, n}^{\prime}=B_{m, n} \tilde{\mathbb{W}}_{m,-n}
$$

И

$$
\bar{G}_{-1 / 2} G_{-1 / 2} \mathbb{U}_{m,-n}=B_{m, n}^{-1} \bar{\partial} \partial \mathbb{O}_{m, n}^{\prime} \quad(\bmod \mathbb{Q}),
$$

где $B_{m, n}$ - коэффициенты, возникающие в ВУД суперсимметричной теории поля Лиувилля [9]. В четырехточечном случае соотношение (21) позволяет свести интеграл по пространству модулей в общем выражении (17) к граничным интегралам, если одно из полей вырожденно, т.е. $a_{i}=a_{m,-n}$. Явный результат имеет вид (см. [10])

$$
I_{4}\left(a_{m,-n}, a_{1}, a_{2}, a_{3}\right)=\kappa N\left(a_{m,-n}\right)\left\{\sum_{i=1}^{3} \sum_{r, s \in(m, n)} q_{r, s}^{(m, n)}\left(a_{i}\right)+2 m n \lambda_{m, n}\right\} \prod_{i=1}^{3} N\left(a_{i}\right)
$$


где

$$
q_{r, s}^{(m, n)}(a)=\left|a-\lambda_{r, s}-\frac{Q}{2}\right|-\lambda_{m, n}
$$

и $(m, n)=\{1-m: 2: m-1,1-n: 2: n-1\}$. Коэффициент равен

$$
\kappa=-2 \mu^{-1} b^{-2}\left[\pi \mu \gamma\left(\frac{1}{2}+\frac{b^{2}}{2}\right)\right]^{2+b^{-2}} \gamma\left[\frac{b^{-2}}{2}-\frac{1}{2}\right],
$$

а множители

$$
N(a)=\left[\pi \mu \gamma\left(\frac{1}{2}+\frac{b^{2}}{2}\right)\right]^{-a / b}\left[\gamma\left(a b-\frac{b^{2}}{2}+\frac{1}{2}\right) \gamma\left(\frac{a}{b}-\frac{b^{-2}}{2}+\frac{1}{2}\right)\right]^{1 / 2} .
$$

\section{4. ПРЯМОЕ ВЫЧИСЛЕНИЕ}

Проверим аналитический результат (22). Пространство параметров $\left(a_{1}, a_{2}, a_{3}, b\right)$ слишком велико, чтобы провести исчерпывающий анализ. Ниже мы сосредоточимся на двух примерах, где одно из полей есть или $\mathbb{W}_{b}$, или $\mathbb{W}_{2 b}$. Более того, ограничимся рассмотрением наиболее симметричного случая четырех тождественных полей $\mathcal{I}_{4}(a)=I_{4}(a, a, a, a)$. В четырехточечном случае выражение (17) сводится к

$$
\mathcal{I}_{4}(a)=\int d^{2} z\left\langle\bar{G}_{-1 / 2} G_{-1 / 2} \mathbb{U}_{a}(z) \mathbb{W}_{a}(0) \tilde{\mathbb{W}}_{a}(1) \mathbb{W}_{a}(\infty)\right\rangle
$$

В данном случае общее выражение (22) дает

$$
\mathcal{I}_{4}(b)=\frac{\kappa}{b} N^{4}(b) \Sigma^{(1,1)}(b),
$$

где

$$
\Sigma^{(1,1)}(b)=\left|2 b^{2}-1\right|
$$

Для второго интеграла имеем

$$
\mathcal{I}_{4}(2 b)=\frac{\kappa}{b} N^{4}(2 b) \Sigma^{(1,3)}(b),
$$

где

$$
\Sigma^{(1,3)}(b)=\frac{3}{2}\left[\left|5 b^{2}-1\right|+\left|3 b^{2}-1\right|+\left|b^{2}-1\right|-3 b^{2}-1\right] .
$$

Рассмотрим интеграл $\mathcal{I}_{4}(b)$. Учитывая, что в этом случае в материальном секторе мы имеем дело с единичными операторами, получаем

$$
\begin{gathered}
\bar{G}_{-1 / 2} G_{-1 / 2} \mathbb{U}_{b}=W_{b}, \\
\mathbb{W}_{b}=V_{b} \bar{c} c \delta(\bar{\gamma}) \delta(\gamma) \\
\tilde{\mathbb{W}}_{b}=\left(\bar{G}_{-1 / 2}^{\mathrm{M}+\mathrm{L}}+\frac{1}{2} \bar{G}_{-1 / 2}^{\mathrm{G}}\right)\left(G_{-1 / 2}^{\mathrm{M}+\mathrm{L}}+\frac{1}{2} G_{-1 / 2}^{\mathrm{G}}\right) V_{b} \bar{c} c .
\end{gathered}
$$


Учитывая явный вид корреляционных функций в духовом секторе,

$$
\langle C(0) C(1)\rangle=0, \quad\langle C(0) C(1) C(\infty)\rangle=1, \quad\langle\delta(\gamma(0)) \delta(\gamma(1))\rangle=1,
$$

приходим к выводу, что единственный ненулевой вклад обусловлен членом в $\tilde{\mathbb{W}}_{b}$, который пропорционален $\bar{c} c$,

$$
\mathcal{I}_{4}(b)=\int d^{2} z\left\langle W_{b}(z) V_{b}(0) W_{b}(1) V_{b}(\infty)\right\rangle .
$$

Таким же образом для второго интеграла имеем

$$
\begin{aligned}
\bar{G}_{-1 / 2} G_{-1 / 2} \mathbb{U}_{2 b} & =\bar{G}_{-1 / 2}^{\mathrm{M}+\mathrm{L}} G_{-1 / 2}^{\mathrm{M}+\mathrm{L}} \Phi_{b} V_{2 b}, \\
\mathbb{W}_{2 b} & =\Phi_{b} V_{2 b} \bar{c} c \delta(\bar{\gamma}) \delta(\gamma), \\
\tilde{\mathbb{W}}_{2 b} & =\left(\bar{G}_{-1 / 2}^{\mathrm{M}+\mathrm{L}}+\frac{1}{2} \bar{G}_{-1 / 2}^{\mathrm{G}}\right)\left(G_{-1 / 2}^{\mathrm{M}+\mathrm{L}}+\frac{1}{2} G_{-1 / 2}^{\mathrm{G}}\right) \Phi_{b} V_{2 b} \bar{c} c
\end{aligned}
$$

а с учетом (32) получаем

$$
\begin{aligned}
\mathcal{I}_{4}(2 b)= & \int d^{2} z\left(\left\langle\Psi_{b}(z) \Phi_{b}(0) \Psi_{b}(1) \Phi_{b}(\infty)\right\rangle\left\langle V_{2 b}(z) V_{2 b}(0) V_{2 b}(1) V_{2 b}(\infty)\right\rangle+\right. \\
& +\left\langle\chi_{b}(z) \Phi_{b}(0) \chi_{b}(1) \Phi_{b}(\infty)\right\rangle\left\langle\bar{Y}_{2 b}(z) V_{2 b}(0) \bar{Y}_{2 b}(1) V_{2 b}(\infty)\right\rangle+ \\
& +\left\langle\bar{\chi}_{b}(z) \Phi_{b}(0) \bar{\chi}_{b}(1) \Phi_{b}(\infty)\right\rangle\left\langle Y_{2 b}(z) V_{2 b}(0) Y_{2 b}(1) V_{2 b}(\infty)\right\rangle+ \\
& \left.+\left\langle\Phi_{b}(z) \Phi_{b}(0) \Phi_{b}(1) \Phi_{b}(\infty)\right\rangle\left\langle W_{2 b}(z) V_{2 b}(0) W_{2 b}(1) V_{2 b}(\infty)\right\rangle\right)
\end{aligned}
$$

Теперь используем симметрию интегралов относительно модулярных преобразований для сведения интегрирования по всей комплексной плоскости к интегрированию по фундаментальной области. Модулярная подгруппа проективных преобразований разбивает комплексную плоскость на шесть областей. Фундаментальная область определяется как $\mathbf{G}=\{\operatorname{Re} x<1 / 2 ;|1-x|<1\}$. Остальные пять областей отображаются в фундаментальную с использованием преобразований $\mathcal{A}, \mathcal{B}, \mathcal{A B}, \mathcal{B} \mathcal{A}$, $\mathcal{A B} \mathcal{A}$, где $\mathcal{A}: z \rightarrow 1 / z$ и $\mathcal{B}: z \rightarrow 1-z$. Комбинируя проективные преобразования полей и соответствующую замену переменных в интегралах, мы сводим интегрирование к фундаментальной области. Заметим, что якобиан преобразования точно сокращает преобразование полей, поскольку их полная конформная размерность равна единице. Тогда

$$
\begin{aligned}
\mathcal{I}_{4}(b)= & 2 \int_{\mathbf{G}} d^{2} z\left(\left\langle W_{b}(z) V_{b}(0) W_{b}(1) V_{b}(\infty)\right\rangle+\left\langle W_{b}(z) V_{b}(0) V_{b}(1) W_{b}(\infty)\right\rangle+\right. \\
& \left.+\left\langle W_{b}(z) W_{b}(0) V_{b}(1) V_{b}(\infty)\right\rangle\right)
\end{aligned}
$$

где множитель 2 перед интегралом учитывает эквивалентные проективные образы. Выражение для второго интеграла оказывается достаточно громоздким:

$$
\mathcal{I}_{4}(2 b)=2 \int_{\mathbf{G}} d^{2} z\left[\left(\left\langle\Psi_{b}(z) \Phi_{b}(0) \Psi_{b}(1) \Phi_{b}(\infty)\right\rangle\left\langle V_{2 b}(z) V_{2 b}(0) V_{2 b}(1) V_{2 b}(\infty)\right\rangle+\right.\right.
$$




$$
\begin{aligned}
& +\left\langle\Psi_{b}(z) \Psi_{b}(0) \Phi_{b}(1) \Phi_{b}(\infty)\right\rangle\left\langle V_{2 b}(z) V_{2 b}(0) V_{2 b}(1) V_{2 b}(\infty)\right\rangle+ \\
& \left.+\left\langle\Psi_{b}(z) \Phi_{b}(0) \Phi_{b}(1) \Psi_{b}(\infty)\right\rangle\left\langle V_{2 b}(z) V_{2 b}(0) V_{2 b}(1) V_{2 b}(\infty)\right\rangle\right)+ \\
& +\left(\left\langle\chi_{b}(z) \Phi_{b}(0) \chi_{b}(1) \Phi_{b}(\infty)\right\rangle\left\langle\bar{Y}_{2 b}(z) V_{2 b}(0) \bar{Y}_{2 b}(1) V_{2 b}(\infty)\right\rangle+\right. \\
& +\left\langle\chi_{b}(z) \chi_{b}(0) \Phi_{b}(1) \Phi_{b}(\infty)\right\rangle\left\langle\bar{Y}_{2 b}(z) \bar{Y}_{2 b}(0) V_{2 b}(1) V_{2 b}(\infty)\right\rangle+ \\
& \left.+\left\langle\chi_{b}(z) \Phi_{b}(0) \Phi_{b}(1) \chi_{b}(\infty)\right\rangle\left\langle\bar{Y}_{2 b}(z) V_{2 b}(0) V_{2 b}(1) \bar{Y}_{2 b}(\infty)\right\rangle\right)+ \\
& +\left(\left\langle\bar{\chi}_{b}(z) \Phi_{b}(0) \bar{\chi}_{b}(1) \Phi_{b}(\infty)\right\rangle\left\langle Y_{2 b}(z) V_{2 b}(0) Y_{2 b}(1) V_{2 b}(\infty)\right\rangle+\right. \\
& +\left\langle\bar{\chi}_{b}(z) \bar{\chi}_{b}(0) \Phi_{b}(1) \Phi_{b}(\infty)\right\rangle\left\langle Y_{2 b}(z) Y_{2 b}(0) V_{2 b}(1) V_{2 b}(\infty)\right\rangle+ \\
& \left.+\left\langle\bar{\chi}_{b}(z) \Phi_{b}(0) \Phi_{b}(1) \bar{\chi}_{b}(\infty)\right\rangle\left\langle Y_{2 b}(z) V_{2 b}(0) V_{2 b}(1) Y_{2 b}(\infty)\right\rangle\right)+ \\
& +\left(\left\langle\Phi_{b}(z) \Phi_{b}(0) \Phi_{b}(1) \Phi_{b}(\infty)\right\rangle\left\langle W_{2 b}(z) V_{2 b}(0) W_{2 b}(1) V_{2 b}(\infty)\right\rangle+\right. \\
& +\left\langle\Phi_{b}(z) \Phi_{b}(0) \Phi_{b}(1) \Phi_{b}(\infty)\right\rangle\left\langle W_{2 b}(z) W_{2 b}(0) V_{2 b}(1) V_{2 b}(\infty)\right\rangle+ \\
& \left.\left.+\left\langle\Phi_{b}(z) \Phi_{b}(0) \Phi_{b}(1) \Phi_{b}(\infty)\right\rangle\left\langle W_{2 b}(z) V_{2 b}(0) V_{2 b}(1) W_{2 b}(\infty)\right\rangle\right)\right]
\end{aligned}
$$

Теперь используем разложение корреляционных функций на конформные блоки. Полезно ввести компактные обозначения. Будем временно опускать некоторые аргументы, которые легко восстановить в окончательных выражениях. В материальном секторе

$$
\begin{aligned}
& \langle\Phi(z) \Phi(0) \Phi(1) \Phi(\infty)\rangle=c_{k}\left|A_{k}^{(0)}(z)\right|^{2} \\
& \langle\Psi(z) \Phi(0) \Psi(1) \Phi(\infty)\rangle=c_{k}\left|A_{k}^{(1)}(z)\right|^{2} \\
& \langle\Psi(z) \Psi(0) \Phi(1) \Phi(\infty)\rangle=c_{k}\left|A_{k}^{(2)}(z)\right|^{2} \\
& \langle\Psi(z) \Phi(0) \Phi(1) \Psi(\infty)\rangle=c_{k}\left|A_{k}^{(3)}(z)\right|^{2} .
\end{aligned}
$$

Здесь индекс $\{k=+, 0,-\}$ соответствует трем каналам в вырожденном операторном произведении поля $\Phi_{b}$ (а также его суперпартнеров), а также предполагается суммирование по $k$. Коэффициенты $c_{k}$ связаны с базисными структурными константами (cм. [10]):

$$
\begin{aligned}
& c_{+}=C_{+}^{2}(b)=\frac{\gamma\left(1 / 2+b^{2} / 2\right) \gamma\left(-1 / 2+5 b^{2} / 2\right)}{\gamma\left(-1 / 2+3 b^{2} / 2\right) \gamma\left(1 / 2+3 b^{2} / 2\right)}, \\
& c_{0}=-\tilde{C}_{0}^{2}(b)=-1, \\
& c_{-}=C_{-}^{2}(b)=-\frac{\gamma\left(2 b^{2}\right) \gamma\left(-1 / 2+b^{2} / 2\right) \gamma^{2}\left(1 / 2+b^{2} / 2\right)}{b^{4} \gamma^{3}\left(b^{2}\right) \gamma\left(-1+b^{2}\right) \gamma\left(-1 / 2+3 b^{2} / 2\right)} .
\end{aligned}
$$

Выражение $A_{k}^{(n)}$ в (38) обозначает конформные блоки, появляющиеся в $k$-м канале для заданной корреляционной функции. Здесь и далее нормировка выбрана так, что все комбинации $c_{k}$, кроме базисных, включены в конформные блоки. Некоторые подробности и явные конструкции, относящиеся к конформным блокам, приведены 
в приложении. В лиувиллевском секторе имеем

$$
\begin{aligned}
\langle V(z) V(0) V(1) V(\infty)\rangle & =\mathcal{R} \int \frac{d P}{4 \pi} r_{l}(P)\left|B_{l}^{(0)}(P, z)\right|^{2} \\
\langle W(z) V(0) W(1) V(\infty)\rangle & =\mathcal{R} \int \frac{d P}{4 \pi} r_{l}(P)\left|B_{l}^{(1)}(P, z)\right|^{2}, \\
\langle W(z) W(0) V(1) V(\infty)\rangle & =\mathcal{R} \int \frac{d P}{4 \pi} r_{l}(P)\left|B_{l}^{(2)}(P, z)\right|^{2}, \\
\langle W(z) V(0) V(1) W(\infty)\rangle & =\mathcal{R} \int \frac{d P}{4 \pi} r_{l}(P)\left|B_{l}^{(3)}(P, z)\right|^{2}
\end{aligned}
$$

Поскольку корреляционные функции не содержат вырожденных полей в лиувиллевском секторе, индекс $l$ здесь предполагает суммирование "четных" и "нечетных" конформных блоков в соответствии с общим операторным произведением (8). Как и ранее, нормировка оставляет только две базисные комбинации вне конформных блоков

$$
\mathcal{R} r_{0}(P)=\mathbb{C}_{a, a}^{Q / 2+i P} \mathbb{C}_{a, a}^{Q / 2-i P}, \quad \mathcal{R} r_{1}(P)=\tilde{\mathbb{C}}_{a, a}^{Q / 2+i P} \tilde{\mathbb{C}}_{a, a}^{Q / 2-i P},
$$

где мы для удобства выделили множитель $\mathcal{R}$ (он не зависит от $P$ ), а параметр $a$ связан с внешней конформной размерностью (т.е. или $b$, или $2 b$ ). Все остальные корреляционные функции в (37) уже не являются независимыми. Они выражаются в терминах тех же основных величин, что и в формулах (38) и (40). Например,

$$
\begin{aligned}
& \langle\chi(z) \Phi(0) \chi(1) \Phi(\infty)\rangle=c_{k} A_{k}^{(1)}(z) A_{k}^{(0)}(\bar{z}), \\
& \langle\chi(z) \chi(0) \Phi(1) \Phi(\infty)\rangle=c_{k} A_{k}^{(2)}(z) A_{k}^{(0)}(\bar{z}) \\
& \langle\chi(z) \Phi(0) \Phi(1) \chi(\infty)\rangle=c_{k} A_{k}^{(3)}(z) A_{k}^{(0)}(\bar{z})
\end{aligned}
$$

И

$$
\begin{aligned}
\langle\bar{Y}(z) V(0) \bar{Y}(1) V(\infty)\rangle & =\mathcal{R} \int \frac{d P}{4 \pi} r_{l}(P) B_{l}^{(0)}(P, z) \bar{B}_{l}^{(1)}(P, \bar{z}), \\
\langle\bar{Y}(z) \bar{Y}(0) V(1) V(\infty)\rangle & =\mathcal{R} \int \frac{d P}{4 \pi} r_{l}(P) B_{l}^{(0)}(P, z) \bar{B}_{l}^{(2)}(P, \bar{z}), \\
\langle\bar{Y}(z) V(0) V(1) \bar{Y}(\infty)\rangle & =\mathcal{R} \int \frac{d P}{4 \pi} r_{l}(P) B_{l}^{(0)}(P, z) \bar{B}_{l}^{(3)}(P, \bar{z}) .
\end{aligned}
$$

Остальные шесть корреляционных функций, не выписанные явно, получаем из (42) и (43) комплексным сопряжением. Используя введенные обозначения, можно переписать рассматриваемые интегралы в компактном виде

$$
\mathcal{I}_{4}(b)=2 \mathcal{R} \int_{\mathbf{G}} d^{2} z \int \frac{d P}{4 \pi} \sum_{l} r_{l}(P)\left[\left|B_{l}^{(1)}(P, z)\right|^{2}+\left|B_{l}^{(2)}(P, z)\right|^{2}+\left|B_{l}^{(3)}(P, z)\right|^{2}\right]
$$

И

$$
\begin{aligned}
\mathcal{I}_{4}(2 b)= & 2 \mathcal{R} \int_{\mathbf{G}} d^{2} z \int \frac{d P}{4 \pi} \sum_{k, l} c_{k} r_{l}(P)\left[\left|A_{k}^{(1)}(z) B_{l}^{(0)}(P, z)+A_{k}^{(0)}(z) B_{l}^{(1)}(P, z)\right|^{2}+\right. \\
& +\left|A_{k}^{(2)}(z) B_{l}^{(0)}(P, z)+A_{k}^{(0)}(z) B_{l}^{(2)}(P, z)\right|^{2}+ \\
& \left.+\left|A_{k}^{(3)}(z) B_{l}^{(0)}(P, z)+A_{k}^{(0)}(z) B_{l}^{(3)}(P, z)\right|^{2}\right] .
\end{aligned}
$$


Громоздкое выражение (37) приобретает замечательно компактную и ясную структуру в терминах конформных блоков. Снова отметим, что в (44) и (45) мы соответственно предполагаем разные значения внешних конформных размерностей $\Delta_{b}$ и $\Delta_{2 b}$ в лиувиллевском секторе.

\section{5. МОДУЛЯРНЫЙ ИНТЕГРАЛ}

При интегрировании оказывается весьма продуктивным [23] использовать эллиптические преобразования. Используем стандартное отображение

$$
\tau=i \frac{K(1-z)}{K(z)},
$$

где полный эллиптический интеграл первого рода есть

$$
K(z)=\frac{1}{2} \int_{0}^{1} \frac{d t}{y},
$$

причем $y^{2}=t(1-t)(1-z t)$. Можно проверить, что

$$
d z=\pi z(1-z) \theta_{3}^{4}(q) d \tau
$$

где $q=e^{i \pi \tau}$ и $\theta_{3}(q)=\sum_{n=-\infty}^{\infty} q^{n^{2}}$. Интеграл (44) принимает вид

$$
\begin{aligned}
\mathcal{I}_{4}(b)= & 2 \pi^{2} \mathcal{R} \int_{-\infty}^{\infty} \frac{d P}{4 \pi} \sum_{l} r_{l}(P)\left[\int_{\mathbf{F}}\left|z(1-z) \theta_{3}^{4}(q) B_{l}^{(1)}(P, z)\right|^{2} d^{2} \tau+\right. \\
& \left.+\int_{\mathbf{F}}\left|z(1-z) \theta_{3}^{4}(q) B_{l}^{(2)}(P, z)\right|^{2} d^{2} \tau+\int_{\mathbf{F}}\left|z(1-z) \theta_{3}^{4}(q) B_{l}^{(3)}(P, z)\right|^{2} d^{2} \tau\right]
\end{aligned}
$$

где $\mathbf{F}=\{|\tau|>1 ;|\operatorname{Re} \tau|<1 / 2\}$. Аналогичным образом для (45) имеем

$$
\begin{aligned}
\mathcal{I}_{4}(2 b)= & 2 \pi^{2} \mathcal{R} \int_{-\infty}^{\infty} \frac{d P}{4 \pi} \sum_{k, l} c_{k} r_{l}(P) \times \\
& \times\left[\int_{\mathbf{F}}\left|z(1-z) \theta_{3}^{4}(q)\left(A_{k}^{(1)}(z) B_{l}^{(0)}(P, z)+A_{k}^{(0)}(z) B_{l}^{(1)}(P, z)\right)\right|^{2} d^{2} \tau+\right. \\
& +\int_{\mathbf{F}}\left|z(1-z) \theta_{3}^{4}(q)\left(A_{k}^{(2)}(z) B_{l}^{(0)}(P, z)+A_{k}^{(0)}(z) B_{l}^{(2)}(P, z)\right)\right|^{2} d^{2} \tau+ \\
& \left.+\int_{\mathbf{F}}\left|z(1-z) \theta_{3}^{4}(q)\left(A_{k}^{(3)}(z) B_{l}^{(0)}(P, z)+A_{k}^{(0)}(z) B_{l}^{(3)}(P, z)\right)\right|^{2} d^{2} \tau\right] .
\end{aligned}
$$

Теперь определим конформные блоки более явно:

$$
\begin{array}{lll}
A_{-}^{(0)}(z)=F_{00}^{\mathrm{M}}(0, z), & A_{0}^{(0)}(z)=F_{01}^{\mathrm{M}}(b, z), & A_{+}^{(0)}(z)=F_{00}^{\mathrm{M}}(2 b, z), \\
A_{-}^{(1)}(z)=F_{11}^{\mathrm{M}}(0, z), & A_{0}^{(1)}(z)=F_{10}^{\mathrm{M}}(b, z), & A_{+}^{(1)}(z)=F_{11}^{\mathrm{M}}(2 b, z), \\
A_{-}^{(2)}(z)=F_{20}^{\mathrm{M}}(0, z), & A_{0}^{(2)}(z)=F_{21}^{\mathrm{M}}(b, z), & A_{+}^{(2)}(z)=F_{20}^{\mathrm{M}}(2 b, z), \\
A_{-}^{(3)}(z)=F_{31}^{\mathrm{M}}(0, z), & A_{0}^{(3)}(z)=F_{30}^{\mathrm{M}}(b, z), & A_{+}^{(3)}(z)=F_{31}^{\mathrm{M}}(2 b, z) .
\end{array}
$$


Здесь первый аргумент симметричных конформных блоков определяет внутреннюю конформную размерность. Первый нижний индекс соответствует одному из четырех базисных типов рассматриваемых конформных блоков по отношению к набору внешних полей (см. приложение); второй индекс равен нулю, если соответствующий блок с данной внутренней конформной размерностью является "четным," и единице, если он "нечетный." В лиувиллевском секторе

$$
\begin{array}{ll}
B_{0}^{(0)}(z)=F_{00}^{\mathrm{L}}(P, z), & B_{1}^{(0)}(z)=F_{01}^{\mathrm{L}}(P, z), \\
B_{0}^{(1)}(z)=F_{11}^{\mathrm{L}}(P, z), & B_{1}^{(1)}(z)=F_{10}^{\mathrm{L}}(P, z), \\
B_{0}^{(2)}(z)=F_{20}^{\mathrm{L}}(P, z), & B_{1}^{(2)}(z)=F_{21}^{\mathrm{L}}(P, z), \\
B_{0}^{(3)}(z)=F_{31}^{\mathrm{L}}(P, z), & B_{1}^{(3)}(z)=F_{30}^{\mathrm{L}}(P, z) .
\end{array}
$$

Для первого интеграла сложные выражения для структурной константы Лиувилля дают

$$
\mathcal{R}=\left(\pi \mu \gamma\left(\frac{b Q}{2}\right) b^{1-b^{2}}\right)^{Q / b-4} \Upsilon_{b}^{6}(b) \Upsilon_{b}^{2}\left(\frac{Q}{2}\right) \Upsilon_{b}^{4}\left(\frac{Q}{2}+b\right)
$$

Специальная функция $\Upsilon_{b}(x)$ является стандартным элементом теории поля Лиувилля (см. в [19] ее определение и свойства). В явном виде имеем

$$
\begin{aligned}
\mathcal{R}= & \left(\pi \mu \gamma\left(\frac{b Q}{2}\right) b^{1-b^{2}}\right)^{Q / b-4} \gamma^{4}\left(\frac{b Q}{2}\right) b^{-4 b^{2}} \times \\
& \times \exp \left\{\int_{0}^{\infty} \frac{d t}{t}\left[\frac{3\left(1-b^{2}\right)^{2} e^{-t}}{2 b^{2}}-\frac{6 \operatorname{sh}^{2}\left(\left(1-b^{2}\right) t / 4 b\right)}{\operatorname{sh}(t / 2 b) \operatorname{sh}(b t / 2)}\right]\right\},
\end{aligned}
$$

где для извлечения дополнительного множителя $(\gamma(b Q / 2))^{4}$ использованы соотношения "сдвига" (см. [19]) для последней ипсилон-функции. Это позволяет улучшить точность сравнения с аналитическим результатом, который содержит тот же самый множитель с сингулярностью при $b=1$. Зависящие от $P$ части равны

$$
\begin{aligned}
& r_{0}(P)=\frac{P^{2} \Upsilon_{b}(b \pm i P) \Upsilon_{b}(Q / 2 \pm i P)}{\Upsilon_{b}^{2}(b-Q / 4 \pm i P / 2) \Upsilon_{b}^{2}(Q / 4 \pm i P / 2) \Upsilon_{b}^{2}(b+Q / 4 \pm i P / 2) \Upsilon_{b}^{2}(3 Q / 4 \pm i P / 2)} \\
& r_{1}(P)=\frac{4 P^{2} \Upsilon_{b}(b \pm i P) \Upsilon_{b}(Q / 2 \pm i P)}{\Upsilon_{b}^{2}(3 b / 2-Q / 4 \pm i P / 2) \Upsilon_{b}^{4}(b / 2+Q / 4 \pm i P / 2) \Upsilon_{b}^{2}(1 / 2 / b+Q / 4 \pm i P / 2)},
\end{aligned}
$$

где введено обозначение $\Upsilon_{b}(x \pm y)=\Upsilon_{b}(x+y) \Upsilon_{b}(x-y)$, а также вновь использованы сдвиговые соотношения для переноса аргументов ипсилон-функций внутрь 
полосы $[0, Q]$, в которой применимо стандартное интегральное представление

$$
\begin{aligned}
& r_{0}(P)=P^{2} \exp \left\{\int _ { 0 } ^ { \infty } \frac { d t } { t } \left[\frac{\left(5-2 b^{2}+5 b^{4}\right)^{2} e^{-t}}{2 b^{2}}+\right.\right. \\
& \left.\left.+\frac{2 \cos \left(\frac{P t}{2}\right)\left(\operatorname{ch} \frac{\left(1-3 b^{2}\right) t}{4 b}+2 \operatorname{ch} \frac{\left(1+b^{2}\right) t}{4 b}+\operatorname{ch} \frac{\left(3-b^{2}\right) t}{4 b}\right)-\cos (P t)\left(\operatorname{ch} \frac{\left(1-b^{2}\right) t}{2 b}+1\right)-6}{\operatorname{sh} \frac{t}{2 b} \operatorname{sh} \frac{b t}{2}}\right]\right\}, \\
& r_{1}(P)=4 P^{2} \exp \left\{\int _ { 0 } ^ { \infty } \frac { d t } { t } \left[-\frac{5\left(1-b^{2}\right)^{2} e^{-t}}{2 b^{2}}+\right.\right. \\
& \left.\left.+\frac{2 \cos \frac{P t}{2}\left(3 \operatorname{ch} \frac{\left(1-b^{2}\right) t}{4 b}+\operatorname{ch} \frac{3\left(1-b^{2}\right) t}{4 b}\right)-\cos (P t)\left(\operatorname{ch} \frac{\left(1-b^{2}\right) t}{2 b}+1\right)-6}{\operatorname{sh} \frac{t}{2 b} \operatorname{sh} \frac{b t}{2}}\right]\right\} .
\end{aligned}
$$

Для второго интеграла аналогично находим явные выражения

$$
\begin{aligned}
\mathcal{R}= & \left(\pi \mu \gamma\left(\frac{b Q}{2}\right) b^{1-b^{2}}\right)^{Q / b-8} \gamma^{4}\left(b^{2}\right) \gamma^{4}\left(\frac{b Q}{2}\right) \gamma^{4}\left(b^{2}+\frac{b Q}{2}\right) b^{4\left(1-6 b^{2}\right)} \times \\
& \times \exp \left\{\int_{0}^{\infty} \frac{d t}{t}\left[\frac{3\left(1-b^{2}\right)^{2} e^{-t}}{2 b^{2}}-\frac{6 \operatorname{sh}^{2} \frac{\left(1-b^{2}\right) t}{4 b}}{\operatorname{sh} \frac{t}{2 b} \operatorname{sh} \frac{b t}{2}}\right]\right\}
\end{aligned}
$$

И

$$
\begin{aligned}
& r_{0}(P)=P^{2} \exp \left\{\int _ { 0 } ^ { \infty } \frac { d t } { t } \left[-\frac{\left(5-18 b^{2}+37 b^{4}\right) e^{-t}}{2 b^{2}}+\right.\right. \\
& \left.\left.+\frac{2 \cos \frac{P t}{2}\left(\operatorname{ch} \frac{\left(1-7 b^{2}\right) t}{4 b}+2 \operatorname{ch} \frac{\left(1+b^{2}\right) t}{4 b}+\operatorname{ch} \frac{\left(3-5 b^{2}\right) t}{4 b}\right)-\cos (P t)\left(\operatorname{ch} \frac{\left(1-b^{2}\right) t}{2 b}\right)+1-6}{\operatorname{sh} \frac{t}{2 b} \operatorname{sh} \frac{b t}{2}}\right]\right\}, \\
& r_{1}(P)=4 P^{2} \exp \left\{\int _ { 0 } ^ { \infty } \frac { d t } { t } \left[-\frac{\left(5-26 b^{2}+37 b^{4}\right) e^{-t}}{2 b^{2}}+\right.\right. \\
& \left.\left.+\frac{2 \cos \frac{P t}{2}\left(\operatorname{ch} \frac{\left(3-7 b^{2}\right) t}{4 b}+2 \operatorname{ch} \frac{\left(1-b^{2}\right) t}{4 b}+\operatorname{ch} \frac{\left(1-5 b^{2}\right) t}{4 b}\right)-\cos P t\left(\operatorname{ch} \frac{\left(1-b^{2}\right) t}{2 b}+1\right)-6}{\operatorname{sh} \frac{t}{2 b} \operatorname{sh} \frac{b t}{2}}\right]\right\} .
\end{aligned}
$$

Конформные блоки можно вычислить, используя численный алгоритм, основанный на рекуррентных соотношениях, найденных в работах [14], [24]-[28]. Мы здесь не используем эллиптическую рекурсию для построения необходимых конформных блоков. Оказывается, что для достижения убедительной точности результатов необходимо знать достаточно небольшое число первых членов в $q$-разложении конформных блоков. Эту информацию можно получить непосредственно используя определение конформных блоков в терминах векторов цепочки (см. приложение) и применяя эллиптическое преобразование. Тем не менее эллиптическое представление (и в особенности вид префактора, т.е. $\Delta$-асимптотики конформных блоков; см. [24]) оказывается очень полезным. Оно позволяет проверить явные выражения для корреляционных функций, удостовериться в выполнении кроссинг-симметрии и, в частности, зафиксировать все знаки, что непросто сделать исходя из общих принципов. 


\section{6. ЧИСЛЕННЫЙ АНАЛИЗ}

С учетом (49) и (50) вычисление сводится к численному интегрированию нескольких интегралов, имеющих общий вид

$$
\int_{\mathbf{F}}\left|z(1-z) \theta_{3}^{4}(q) \mathcal{F}_{P}(z)\right|^{2} d^{2} \tau
$$

где $\mathcal{F}_{P}(z)$ - некоторый лиувиллевский конформный блок, подобный приведенному в (49), или некоторое более сложное составное выражение, подобное (50). Подынтегральное выражение можно разложить в двойной степенной ряд по $q$ и $\bar{q}$ в соответствии с общим разложением

$$
z(1-z) \theta_{3}^{4}(q) \mathcal{F}_{P}(z)=(16 q)^{\alpha} \sum_{r=0}^{\infty} b_{r}(P) q^{r},
$$

где $\alpha$ и коэффициенты $b_{r}$ определяются конкретным выбором функции $\mathcal{F}_{P}(z)$. В каждом слагаемом можно явно проинтегрировать по $\tau_{2}=\operatorname{Im} \tau$, результат выражается через функцию

$$
\begin{aligned}
\Phi(A, r, l) & =\int_{\mathbf{F}}|16 q|^{2 A} q^{r} \bar{q}^{l} d^{2} \tau= \\
& =\frac{(16)^{2 A}}{\pi(2 A+r+l)} \int_{-1 / 2}^{1 / 2} \cos (\pi(r-l) x) e^{-\pi \sqrt{1-x^{2}}(2 A+r+l)} d x .
\end{aligned}
$$

С учетом (49) мы имеем сумму шести интегралов вида (59) и получаем ряд

$$
\mathcal{I}_{4}(b)=\pi \mathcal{R} \sum_{L=0}^{\infty}\left(A_{L}^{(1, \mathrm{e})}+A_{L}^{(1, \mathrm{o})}+A_{L}^{(2, \mathrm{e})}+A_{L}^{(2, \mathrm{o})}+A_{L}^{(3, \mathrm{e})}+A_{L}^{(3, \mathrm{o})}\right),
$$

где

$$
\begin{aligned}
& A_{L}^{(1, \mathrm{e})}=\int_{0}^{\infty} r_{2}(P) d P \sum_{k=0}^{L} b_{k}^{(1, \mathrm{e})}(P) b_{L-k}^{(1, \mathrm{e})}(P) \Phi\left(\frac{P^{2}}{2}+\frac{Q^{2}}{8}-\frac{1}{2}, k, L-k\right), \\
& A_{L}^{(1, \mathrm{o})}=\int_{0}^{\infty} r_{1}(P) d P \sum_{k=0}^{L} b_{k}^{(1, \mathrm{o})}(P) b_{L-k}^{(1, \mathrm{o})}(P) \Phi\left(\frac{P^{2}}{2}+\frac{Q^{2}}{8}, k, L-k\right), \\
& A_{L}^{(2, \mathrm{e})}=\int_{0}^{\infty} r_{2}(P) d P \sum_{k=0}^{L} b_{k}^{(2, \mathrm{e})}(P) b_{L-k}^{(2, \mathrm{e})}(P) \Phi\left(\frac{P^{2}}{2}+\frac{Q^{2}}{8}-\frac{1}{2}, k, L-k\right), \\
& A_{L}^{(2, \mathrm{o})}=\int_{0}^{\infty} r_{1}(P) d P \sum_{k=0}^{L} b_{k}^{(2, \mathrm{o})}(P) b_{L-k}^{(2, \mathrm{o})}(P) \Phi\left(\frac{P^{2}}{2}+\frac{Q^{2}}{8}, k, L-k\right), \\
& A_{L}^{(3, \mathrm{e})}=\int_{0}^{\infty} r_{1}(P) d P \sum_{k=0}^{L} b_{k}^{(3, \mathrm{e})}(P) b_{L-k}^{(3, \mathrm{e})}(P) \Phi\left(\frac{P^{2}}{2}+\frac{Q^{2}}{8}-1, k, L-k\right), \\
& A_{L}^{(3, \mathrm{o})}=\int_{0}^{\infty} r_{2}(P) d P \sum_{k=0}^{L} b_{k}^{(3, \mathrm{o})}(P) b_{L-k}^{(3, \mathrm{o})}(P) \Phi\left(\frac{P^{2}}{2}+\frac{Q^{2}}{8}-\frac{1}{2}, k, L-k\right) .
\end{aligned}
$$

Каждое слагаемое в (63) подавлено фактором $\max _{\mathbf{F}}|q|^{2 L}$, так что ряд по $L$ сходится в численных расчетах очень быстро. Мы нашли, что для достижения точности в 


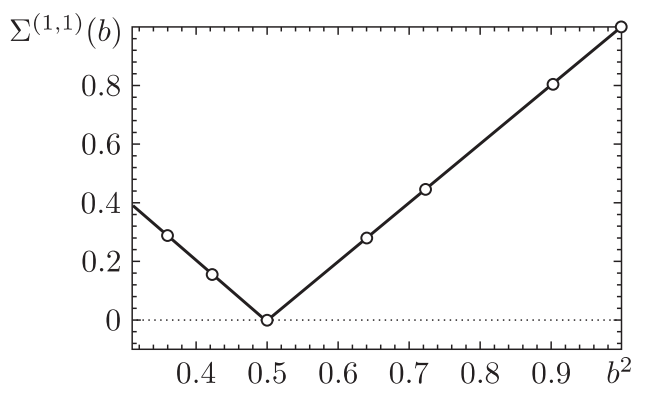

a

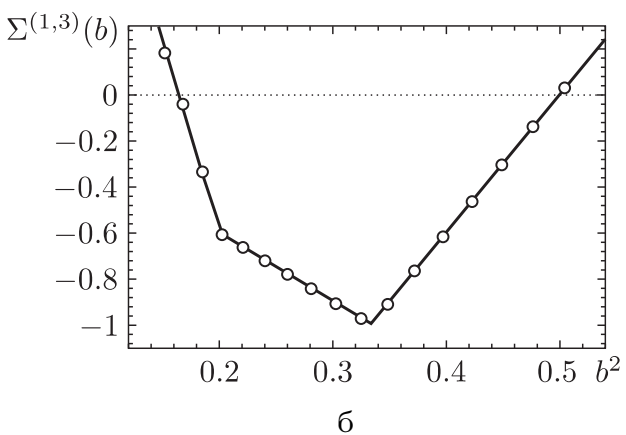

б

Прямое численное вычисление редуцированных интегралов (27) (а) и (29) (б)

(точки) в сравнении с точной формулой (сплошная линия).

три или четыре значащие цифры достаточно суммировать до $L=4$ (см. табл. 1). Во втором случае мы численно интегрируем таким же образом, хотя теперь имеется 18 интегралов вида (59). В этом случае суммирование до $L=4$ позволило достичь точности в две или три значащие цифры (см. табл. 2). На рисунке результаты численного вычисления интегралов (27) и (29) показаны в виде окружностей, а линии соответствуют точному результату.

ТАБлицА 1. Численные данные для $\Sigma^{(1,1)}(b)$ при $\mu=1$

\begin{tabular}{|c|c|c|}
\hline$b$ & Численное значение & Точное значение \\
\hline 0.999 & 0.9959 & 0.9960 \\
0.95 & 0.8049 & 0.8050 \\
0.85 & 0.4450 & 0.4450 \\
0.80 & 0.2799 & 0.2800 \\
$1 / \sqrt{2}$ & 0.0001 & 0 \\
0.65 & 0.1555 & 0.1550 \\
0.60 & 0.2877 & 0.2800 \\
\hline
\end{tabular}

ТАБлицА 2. Численные данные для $\Sigma^{(1,3)}(b)$ при $\mu=1$

\begin{tabular}{|c|c|c|}
\hline$b$ & Численное значение & Точное значение \\
\hline 0.71 & 0.0309 & 0.0246 \\
0.69 & -0.1377 & -0.1434 \\
0.67 & -0.3030 & -0.3066 \\
0.65 & -0.4623 & -0.4650 \\
0.63 & -0.6159 & -0.6186 \\
0.61 & -0.7644 & -0.7674 \\
0.59 & -0.9096 & -0.9114 \\
0.57 & -0.9699 & -0.9747 \\
0.55 & -0.9060 & -0.9075 \\
0.53 & -0.8409 & -0.8427 \\
0.51 & -0.7791 & -0.7803 \\
0.49 & -0.7197 & -0.7203 \\
0.47 & -0.6621 & -0.6627 \\
0.45 & -0.6069 & -0.6075 \\
0.43 & -0.3342 & -0.3282 \\
0.41 & -0.0384 & -0.0258 \\
0.39 & 0.1827 & 0.2622 \\
\hline
\end{tabular}




\section{ПРИЛОЖЕНИЕ}

\section{Конформные блоки и векторы цепочки}

Для определенности будем использовать обозначения лиувиллевского сектора, хотя результаты, относящиеся к конформному блоку, универсальны (т.е. не зависят от сектора). Схематично вклад данного конформного семейства в четырех основных операторных разложениях можно записать в виде

$$
\begin{gathered}
V_{1}(z) V_{2}(0)=z^{\Delta-\Delta_{1}-\Delta_{2}} \sum_{N} z^{N}|N\rangle_{12}, \\
W_{1}(z) V_{2}(0)=z^{\Delta-\Delta_{1}-\Delta_{2}-1 / 2} \sum_{N} z^{N} \widetilde{N\rangle}_{12}, \\
V_{1}(z) W_{2}(0)=z^{\Delta-\Delta_{1}-\Delta_{2}-1 / 2} \sum_{N} z^{N} \widetilde{\widetilde{|N\rangle}} \\
W_{1}(z) W_{2}(0)=z^{\Delta-\Delta_{1}-\Delta_{2}-1} \sum_{N} z^{N} \widetilde{\widetilde{|N\rangle}}_{12},
\end{gathered}
$$

где так называемые векторы цепочки $|N\rangle, \widetilde{|N\rangle}, \widetilde{\widetilde{|N\rangle}} \underset{\widetilde{|N\rangle}}{\approx}$ (с положительным целым или полуцелым $N$ ) являются вкладом потомков $N$-го уровня промежуточного состояния с конформной размерностью $\Delta$, возникающего в данном операторном произведении. Векторы цепочки полностью определяются суперконформной симметрией. А именно, суперконформные условия приводят к рекуррентным соотношениям

$$
\begin{aligned}
G_{k}|N\rangle_{12} & =|\widetilde{N-k}\rangle_{12}, \\
G_{k} \mid \widetilde{|N\rangle} & =\left[\Delta+2 k \Delta_{1}-\Delta_{2}+N-k\right]|N-k\rangle_{12}
\end{aligned}
$$

при $k>0$ и

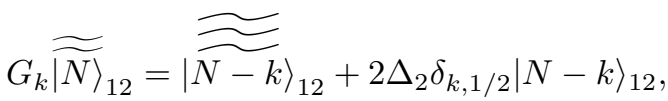

$$
\begin{aligned}
& G_{k}{\widetilde{\widetilde{\widetilde{N}\rangle_{12}}}}_{12}=\left[\Delta+2 k \Delta_{1}-\left(\Delta_{2}+1 / 2\right)+N-k\right]\left|\widetilde{\widetilde{N-k}\rangle_{12}}-2 \Delta_{2} \delta_{k, 1 / 2}\right| \widetilde{N-k\rangle_{12}}
\end{aligned}
$$

также при $k>0$. Нормировка векторов цепочки в данном изложении определяется требованиями

$$
|0\rangle=1, \quad \widetilde{|0\rangle}=1, \quad \widetilde{\widetilde{|0\rangle}}=-1, \quad \stackrel{\widetilde{\widetilde{|0\rangle}}}{\approx}=\left(\Delta-\Delta_{1}-\Delta_{2}\right) .
$$

Соотношения (65) и (66) эквивалентны линейной задаче для коэффициентов, определяющих векторы цепочки через базисные векторы Вирасоро на том же уровне. Эту систему можно решить численно вплоть до достаточно высокого уровня. 
Требуемые суперконформные блоки определяются из разложений

$$
\begin{aligned}
& \mathcal{F}_{\mathrm{e}, \mathrm{o}}\left(\begin{array}{ll}
a_{1} & a_{3} \\
a_{2} & a_{4}
\end{array}|\Delta| z\right)=z^{\Delta-\Delta_{1}-\Delta_{2}} \sum_{N \in \mathbb{Z}, \mathbb{Z} / 2} z^{N}{ }_{12}\langle N \mid N\rangle_{34}, \\
& \mathcal{F}_{\mathrm{e}, \mathrm{o}}\left(\begin{array}{ll}
\hat{a}_{1} & \hat{a}_{3} \\
a_{2} & a_{4}
\end{array}|\Delta| z\right)=z^{\Delta-\Delta_{1}-\Delta_{2}-1 / 2} \sum_{N \in \mathbb{Z}, \mathbb{Z} / 2} z^{N}{ }_{12} \widetilde{\langle N \mid N\rangle_{34}}, \\
& \mathcal{F}_{\mathrm{e}, \mathrm{o}}\left(\begin{array}{ll}
\hat{a}_{1} & a_{3} \\
\hat{a}_{2} & a_{4}
\end{array}|\Delta| z\right)=z^{\Delta-\Delta_{1}-\Delta_{2}-1} \sum_{N \in \mathbb{Z}, \mathbb{Z} / 2} z^{N}{ }_{12} \stackrel{\widetilde{\widetilde{\langle N|}}}{\widetilde{\langle N}\rangle_{34}}, \\
& \mathcal{F}_{\mathrm{e}, \mathrm{o}}\left(\begin{array}{cc}
\hat{a}_{1} & a_{3} \\
a_{2} & \hat{a}_{4}
\end{array}|\Delta| z\right)=z^{\Delta-\Delta_{1}-\Delta_{2}-1 / 2} \sum_{N \in \mathbb{Z}, \mathbb{Z} / 2} z^{N} \widetilde{{ }_{12} \widetilde{\langle N \mid N\rangle}}{ }_{34} .
\end{aligned}
$$

В тексте используются сокращенные обозначения

$$
\begin{array}{ll}
F_{00}(\Delta, z)=\mathcal{F}_{\mathrm{e}}\left(\begin{array}{cc}
a & a \\
a & a
\end{array}|\Delta| z\right), & F_{01}(\Delta, z)=\mathcal{F}_{\mathrm{o}}\left(\begin{array}{cc}
a & a \\
a & a
\end{array}|\Delta| z\right), \\
F_{10}(\Delta, z)=\mathcal{F}_{\mathrm{e}}\left(\begin{array}{cc}
\hat{a} & \hat{a} \\
a & a
\end{array}|\Delta| z\right), & F_{11}(\Delta, z)=\mathcal{F}_{\mathrm{o}}\left(\begin{array}{cc}
\hat{a} & \hat{a} \\
a & a
\end{array}|\Delta| z\right), \\
F_{20}(\Delta, z)=\mathcal{F}_{\mathrm{e}}\left(\begin{array}{cc}
\hat{a} & a \\
\hat{a} & a
\end{array}|\Delta| z\right), & F_{21}(\Delta, z)=\mathcal{F}_{\mathrm{o}}\left(\begin{array}{cc}
\hat{a} & a \\
\hat{a} & a
\end{array}|\Delta| z\right), \\
F_{30}(\Delta, z)=\mathcal{F}_{\mathrm{e}}\left(\begin{array}{cc}
\hat{a} & a \\
a & \hat{a}
\end{array}|\Delta| z\right), & F_{31}(\Delta, z)=\mathcal{F}_{\mathrm{o}}\left(\begin{array}{cc}
\hat{a} & a \\
a & \hat{a}
\end{array}|\Delta| z\right) .
\end{array}
$$

Благодарности. Автор благодарит LPTA в Университете Montpellier II за гостеприимство и стимулирующую атмосферу. Особая благодарность В. А. Фатееву и А. Невё за их воодушевляющий интерес к данной работе. Автор благодарен также А. А. Белавину за полезные обсуждения. Данная работа поддержана РФФИ (гранты № 08-01-00720 и РФФИ-CNRS PICS-09-02-91064). Часть вычислений была выполнена при посещении Математического факультета Университета Киото в январе 2008 года. Автор признателен указанному факультету и лично Т. Миве.

\section{Список литературы}

[1] A. M. Polyakov, Phys. Lett. B, 103:3 (1981), 211-213.

[2] Al. Zamolodchikov, Internat. J. Modern Phys. A, 19, Suppl. 2 (2004), 510-523; arXiv: hep-th/0312279.

[3] А.А. Белавин, А. Б. Замолодчиков, ТМФ, 147:3 (2006), 339-371; arXiv: hep-th/0510214.

[4] A. Belavin, A. B. Zamolodchikov, Писъма в ЖЭЭТФ, 82:1 (2005), 8-14.

[5] P. Ginsparg, G. Moore, Lectures on $2 D$ gravity and $2 D$ string theory (TASI-1992), arXiv: hep-th/9304011; P. Di Francesco, P. Ginsparg, J. Zinn-Justin, Phys. Rep., 254:1-2 (1995), 1-133; arXiv: hep-th/9306153.

[6] A. Belavin, A. Zamolodchikov, J. Phys. A, 42 (2009), 304004; arXiv:0811.0450.

[7] A. A. Belavin, A. M. Polyakov, A. B. Zamolodchikov, Nucl. Phys. B, 241:2 (1984), 333-380.

[8] V. G. Kac, Infinite-Dimensional Lie Algebras, Progr. Math., 44, Birkhäuser, Boston, MA, 1983. 
[9] А. А. Белавин, А. Б. Замолодчиков, Писъма в ЖЭЭФФ, 84:8 (2006), 496-502.

[10] A. Belavin, V. Belavin, J. Phys. A, 42 (2009), 304003; arXiv:0810.1023.

[11] F. David, Modern Phys. Lett. A, 3:17 (1988), 1651-1656.

[12] J. Distler, H. Kawai, Nucl. Phys. B, 231:2 (1989), 509-527.

[13] J. Distler, Z. Hlousek, H. Kawai, Internat. J. Modern Phys. A, 5:2 (1990), 391-414.

[14] A. Belavin, V. Belavin, A. Neveu, Al. Zamolodchikov, Nucl. Phys. B, 784:3 (2007), 202-233; arXiv: hep-th/0703084.

[15] R. H. Poghossian, Nucl. Phys. B, 496:1-2 (1997), 451-464.

[16] R. Rashkov, M. Stanishkov, Phys. Lett. B, 380:1-2 (1996), 49-58.

[17] T. Fukuda, K. Hosomichi, Nucl. Phys. B, 635:1-2 (2002), 215-254; arXiv: hep-th/0202032.

[18] H. Dorn, H.-J. Otto, Phys. Lett. B, 291:1-2 (1992), 39-43; arXiv: hep-th/9206053; Nucl. Phys. B, 429:2 (1994), 375-388; arXiv: hep-th/9403141.

[19] A. Zamolodchikov, Al. Zamolodchikov, Nucl. Phys. B, 477:2 (1996), 577-605.

[20] J. Polchinski, String Theory. V. 2. Superstring Theory and Beyond, Cambridge Monogr. Math. Phys., Cambridge Univ. Press, Cambridge, 1998.

[21] E. Verlinde, H. Verlinde, "Lectures On String Perturbation Theory", Superstrings'88, eds. M. Green, M. Grisaru, R. Iengo, E. Sezgin, A. Strominger, World Sci., Teaneck, NJ, 1989, 189-250.

[22] D. Friedan, JHEP, 10 (2003), 063; arXiv: hep-th/0204131.

[23] Ал. Б. Замолодчиков, ТМФ, 151:3 (2007), 439-457; arXiv: hep-th/0604158.

[24] L. Hadasz, Z. Jaskolski, P. Suchanek, Nucl. Phys. B, 798:3 (2008), 363-378; arXiv: 0711.1619.

[25] L. Hadasz, Z. Jaskólski, P. Suchanek, JHEP, 03 (2007), 032; arXiv: hep-th/0611266.

[26] В. А. Белавин, ТМФ, 152:3 (2007), 476-487; arXiv: hep-th/0611295.

[27] V.A. Belavin, Nucl. Phys. B, 798:3 (2008), 423-442; arXiv:0705.1983.

[28] D. Chorażkiewicz, L. Hadasz, JHEP, 01 (2009), 007; arXiv:0811.1226.

Поступила в редакцию 12.05.2009 